\title{
Deciphering the Anode-Enhanced Azo Dye Degradation in Anaerobic Baffled Reactors Integrating With Microbial Fuel Cells
}

\author{
Yonggang Yang ${ }^{1,2}$, Ou Luo ${ }^{1}$, Guannan Kong ${ }^{1}$, Bin Wang ${ }^{1}$, Xiaojing Li' ${ }^{1}$, Enze Li ${ }^{1}$, \\ Jianjun $\mathrm{Li}^{1,2}$, Feifei $\mathrm{Liu}^{1}$ and Meiying $\mathrm{Xu}^{1,2 *}$
}

' Guangdong Provincial Key Laboratory of Microbial Culture Collection and Application, Guangdong Institute of Microbiology, Guangzhou, China, ${ }^{2}$ State Key Laboratory of Applied Microbiology Southern China, Guangzhou, China

OPEN ACCESS

Edited by:

Yang-Chun Yong,

Jiangsu University, China

Reviewed by:

Jinyou Shen,

Nanjing University of Science

and Technology, China

Krishnaveni Venkidusamy,

University of South Australia, Australia

${ }^{*}$ Correspondence:

Meiying Xu

xumy@gdim.cn

Specialty section:

This article was submitted to Microbiotechnology, Ecotoxicology and Bioremediation,

a section of the journal

Frontiers in Microbiology

Received: 17 May 2018

Accepted: 20 August 2018 Published: 06 September 2018

Citation:

Yang Y, Luo O, Kong G, Wang B,

Li X, Li E, Li J, LiU F and XU M (2018) Deciphering

the Anode-Enhanced Azo Dye Degradation in Anaerobic Baffled Reactors Integrating With Microbial Fuel Cells. Front. Microbiol. 9:2117. doi: 10.3389/fmicb.2018.02117
Microbial anode respiration in microbial fuel cells (MFCs) can enhance the degradations of many electron acceptor-type contaminants which are presumed to be competitive to anode respiration. The mechanisms underlying those counterintuitive processes are important for MFCs application but are unclear. This study integrated MFCs with anaerobic baffled reactor (ABR), termed MFC-ABR, to enhance the reduction of azo dye acid orange-7 (AO-7). Compare with ABR, MFC-ABR enhanced the degradation of $\mathrm{AO}-7$, especially at high $\mathrm{AO}-7$ concentration $(800 \mathrm{mg} / \mathrm{L})$. Acute toxicity test suggested a higher detoxication efficiency in MFC-ABR. Higher microbial viability, dehydrogenase activity and larger sludge granule size were also observed in MFC-ABR. MFC-ABR significantly enriched and reshaped the microbial communities relative to ABR. Bacteria with respiratory versatility, e.g., Pseudomonas, Geobacter, and Shewanella, were significantly enriched. Functional prediction showed that six metabolism functions (manganese-, iron-, fumarate- and nitrate-respiration, oil bioremediation and chemoheterotrophy) were significantly stimulated while methanogenesis, sulfate-respiration, hydrogen-oxidation were suppressed in MFC-ABR relative to $A B R$. The results provided important information for understanding the role of microbial anode respiration in contaminated environments.

Keywords: microbial fuel cells, anode respiration, azo dye, wastewater treatment, anaerobic baffled reactor

\section{INTRODUCTION}

Azo dyes are the most extensively used synthetic dyes in various commercial applications and $10-20 \%$ of them are lost in effluent during dyeing process (Li et al., 2010; Solis et al., 2012; Solanki et al., 2013). Those commercial dyes are generally highly stable in light and resistant to aerobic biodegradation. Wastewaters containing azo dyes or their breakdown products are usually toxic, mutagenic and inhibit light penetration in water bodies, hence affecting aquatic ecosystems (Mu et al., 2009; Sun et al., 2009). Toxication events caused by azo dye containing effluents are increasingly reported (de Aragao Umbuzeiro et al., 2005).

Biodegradation is a widely used methods for azo dye treatment in which the microbial azo reduction under anaerobic condition was considered to be the rate-limiting step (Solis et al., 2012). Many efforts have been made to stimulate the anaerobic azo reduction. Microbial fuel 
cells (MFCs) is a group of bioelectrochemical system (BES) capable of converting the contaminants in wastewater into electricity via microbial extracellular electron transfer (EET) to anode (Solanki et al., 2013; Jiang et al., 2018; Zhao et al., 2018). It has been shown that azo reduction could be enhanced by either anode or cathode in MFCs (Mu et al., 2009; Sun et al., 2009; Solanki et al., 2013). Moreover, by integrating BESs with traditional wastewater treatment reactors, the treatment efficiency and feasibility could be further enhanced compared to the traditional reactors or BESs (Cui et al., 2014; Kong et al., 2015; Xu et al., 2018a).

The cathode-enhanced azo dye reduction in an MFC is expectable as azo dyes can serve as electron acceptors to be reduced electrochemically or biologically by cathode or cathodic biofilms (Mu et al., 2009). However, several reports have shown anode-stimulated azo dye reduction which were counterintuitive as anode, as another electron acceptor for microbes, was assumed to be competitive to microbial azo dye reduction (Sun et al., 2009; Liu et al., 2013; Solanki et al., 2013). In addition to azo dyes, the biodegradation of some other electron acceptortype contaminants [e.g., nitrophenol, chlorinated hydrocarbons and vanadium $(\mathrm{V})$ ] could also be stimulated by MFC anodes (Pham et al., 2009; Hao et al., 2016; Jiang et al., 2018; Xu et al., 2018b; Zhao et al., 2018). Electron acceptors such as oxygen, anode, nitrate, $\mathrm{Fe}(\mathrm{III})$ are usually used to stimulate biodegradations in wastewater treatment or bioremediation. Therefore understanding the anode-enhanced biodegradation of azo dye will be important to MFC application and other electron acceptor-driven biostimulation processes. However, the mechanism underlying such counterintuitive phenomenon is still unclear.

In this study, we integrated MFC electrodes with an anaerobic baffled reactor (ABR), a traditional wastewater treatment reactor to enhance the degradation of a commercial sulfonated azo dye Acid Orange 7 (AO-7). The AO-7 degradation, COD removal and effluent toxicity were evaluated. Moreover, the sludge particle size, microbial viability and dehydrogenase (DHA) activity, microbial community structure and function were comparatively analyzed to decipher the anode-enhanced azo dye degradation in MFC-ABR.

\section{MATERIALS AND METHODS}

\section{Anaerobic Baffled Reactors Coupling With Microbial Fuel Cells (MFC-ABR)}

Each MFC-ABR and ABR reactors were made of plexiglass [353 mm (length) $\times 80 \mathrm{~mm}$ (width) $\times 400 \mathrm{~mm}$ (height)] with an effective volume of $8.4 \mathrm{~L}$ (Supplementary Figure S1). Each reactor contained three chambers which were separated by two vertical over-flow plates. Each compartment is further divided into an up-flow and a down-flow part by a vertical baffle, the volume of the two part is 4:1. The heights of the over-flow plates decreased from 40 to 35 and $30 \mathrm{~cm}$ to allow the influent flows from one compartment to the next. The bottom of each baffle plates have a $45^{\circ}$ slant edge and a distance of $1 \mathrm{~cm}$ to the $\mathrm{ABR}$ inner bottom.
The anode of the MFC module is carbon brush that made by bonding plain carbon fibers (Liso Composite Material, China) to a stainless steel wire $(15 \mathrm{~cm}$ in length and $5 \mathrm{~cm}$ in diameter), while the cathode is a piece of rectangle carbon felt $(5 \times 4 \times 1 \mathrm{~cm}$, Liso Composite Material, China). Both anodes and cathodes were used after being boiled in deionized water for $20 \mathrm{~min}$. The anode was embedded in the sludge in each chamber and the carbon felt cathode was fixed at the water surface.

Six reactors were assembled and contained an anode and a cathode in each chamber. The anodes and cathodes of three reactors was connected by titanium wires with $1000 \mathrm{ohm}$ resistors, i.e., MFC-ABR. The cathodes and anodes in the other three reactors was not connected, i.e., ABR.

\section{Inoculation and Operational Conditions}

The ABR and MFC-ABR was inoculated with sludge from a secondary settling tank at Liede wastewater plant (Guangzhou, China). Before inoculation, the sludge was stored in a bucket at room temperature $\left(23 \pm 3^{\circ} \mathrm{C}\right)$ for 1 day. Then, the overlying water was removed and the sludge was added into the ABR and MFC-ABR reactors. Each chamber was inoculated with $1.1 \mathrm{~L}$ of sludge to embed the anode.

Medium for all reactors contained (per Liter): $0.41 \mathrm{~g}$ $\mathrm{CH}_{3} \mathrm{COONa}, 0.407 \mathrm{~g} \mathrm{NH} 4 \mathrm{Cl}, 6 \mathrm{~g} \mathrm{Na}_{2} \mathrm{HPO}_{4}, 3$ g $\mathrm{KH}_{2} \mathrm{PO}_{4}$, $0.5 \mathrm{~g} \mathrm{NaCl}, 0.1 \mathrm{~g} \mathrm{MgSO}_{4} \cdot 7 \mathrm{H}_{2} \mathrm{O}, 0.015 \mathrm{~g} \mathrm{CaCl}_{2} \cdot 7 \mathrm{H}_{2} \mathrm{O}$. And the concentration of AO-7 was increased from 200 (stage I, 27 days) to 400 (stage II, 28 days) and 800 (stage III, 25 days) mg/L to evaluate the performances of $\mathrm{ABR}$ and MFC-ABR under different azo dye concentrations. The medium was pumped into all reactors with peristaltic pumps with a hydraulic detention time of $24 \mathrm{~h}$. The experiments were conducted at ambient temperature.

\section{Chemical Analyses}

The effluent for chemical analyses were sampled every 3 days. The samples were centrifuged with $8000 \mathrm{rpm}$ for $5 \mathrm{~min}$, then filtered through $0.45 \mu \mathrm{m}$-pore-size syringe filter. AO-7 concentration of the effluent was measured with an UV-visible spectrophotometer (UV-2600, SHIMADZU) at $484 \mathrm{~nm}$. The reduced products of AO-7 degradation were identified and quantified with high performance liquid chromatography (HPLC). The HPLC system used a Zorbax SB-C18 $(150 \mathrm{~mm} \times 4.6 \mathrm{~mm} \times 5 \mu \mathrm{m}$, Agilent $)$ for separation and a UV detector for measurement at $254 \mathrm{~nm}$ (Thung et al., 2018). COD in the effluents was pretreated with a $\mathrm{HACH}$ fast digester (HACH 2800) and measured at $600 \mathrm{~nm}$ using an UV-visible detector. The particle diameter of the sludge was analyzed using a particle size and shape analyzer (Ankersmid Eyetech) with an analyzing range of $0.1-3600 \mu \mathrm{m}$. The Coulombic efficiencies of each chamber in different stages were calculated as previously reported (Logan et al., 2006).

\section{Effluent Eco-Toxicity Test}

Acute eco-toxicity of the effluents was tested as reported before (Yin et al., 2005). Briefly, $30 \mathrm{~mL}$ of effluent samples were collected from the three chambers of each reactor at the end of the experiments. Prior to measurement, samples were centrifuged at $4000 \mathrm{rpm}$ for $5 \mathrm{~min}$. The supernatant $(20 \mathrm{ml})$ was added into a beaker that contains eight Daphnia magna neonates born within 
$24 \mathrm{~h}$. The number of living neonates was recorded after $24 \mathrm{~h}$. The survival rate of the Daphnia magna was used to evaluate the acute toxicity of the effluents. Three replicates were done for each effluent samples.

\section{Biomass and Microbial Activity Analyses}

The sludge and anodes were sampled from each reactor at the end of experiment. The biofilm-covered anode brushes were rinsed in sterilized phosphate buffer $(\mathrm{pH}=7.0)$ to remove loosely attached bacteria cells and sludge residues. The protein-based biomass of the sludge was analyzed by a Bradford-protein quantification kit (PA102, TIANGEN). DHA activity was tested by evaluating the 2, 3, 5 - triphenyl tetrazoliumchloride (TTC) reducing capacity of the sludge samples at a wavelength of $485 \mathrm{~nm}$ by a UV-visible spectrophotometer (UV-2600, SHIMADZU) (Bozich et al., 2017). The microbial metabolism activity in sludge and anode biofilms were analyzed using a confocal laser scanning microscopy (CLSM, LSM 700) after being stained by LIVE/DEAD BacLight staining kit (Molecular Probes, Invitrogen), as described in our previous report (Yang et al., 2015). The specific viability (r) of the sludge and biofilms was presented as the ratio of viable to total biofilm cells based on pixel counting (Yang et al., 2015).

\section{DNA Sequencing and Community Analyses}

After stage-III, the sludge samples in different chambers were collected. $10 \mathrm{ml}$ of each sludge sample were centrifuged at $5000 \mathrm{rpm}$ for $10 \mathrm{~min}$. DNA of the collected precipitates were was extracted using a PowerSoil ${ }^{\circledR}$ DNA Isolation Kit for Soil (Mo Bio Laboratories Inc.). Amplification of the $16 \mathrm{~S}$ rRNA gene V4 region was done using the primers of 515F (5'-GTGCCAGCMGCCGCGGTAA-3') and 806R (5'-GGACTACHVGGGTWTCTAAT-3') with Illumina adapters. The DNA amplification, sequence processing and statistical analysis were conducted as described in our previous reports ( $\mathrm{Xu}$ et al., 2014; Sun et al., 2017). The NCBI accession number to the sequence data is PRJNA479585. Before analyses, the sequence data were processed using QIIME Pipeline-Version 1.7.0. All sequence reads were trimmed and assigned to each sample based on their barcodes. Sequences $<200$ bp were removed with an average base quality score $\mathrm{Q}<25$. The $16 \mathrm{~S}$ rRNA gene sequences were used for chimera check using the Uchime algorithm. Sequences were clustered into operational taxonomic units (OTUs) using a 97\% identity threshold. Each sample was rarefied to the sample exhibiting the lowest number of reads for both alpha-diversity (observed species, Shannon's and Simpson's diversity index) and beta diversity (PCoA, UniFrac) analyses, for which the rarefaction curves were generated from the observed species. The principal co-ordinates analysis (PCoA) based on the Bray-Curties distance were executed to investigate the relationships among all samples. The redundancy analyses (RDA) were applied to explore the relationships between the samples and environmental variables including $\mathrm{pH}$, dissolved oxygen (DO), oxidation-reduction potential (ORP), voltage and AO-7 removal efficiency. Both of the PCoA and RDA were executed by applying the vegan packages in $\mathrm{R}$ software. Taxonomy was assigned using the Ribosomal Database Project classifier at a confidence-level of $80 \%$. OTUs with the abundances exceeding $1 \%$ were selected to compare the bacterial communities from different samples unless stated otherwise. FAPROTAX database was used to evaluate the functional composition of the OTUs in the sludge from different reactors (Louca et al., 2016).

TABLE 1 | Genera (abundance $>0.1 \%$ ) with significantly different abundances between MFC-ABR and ABR.

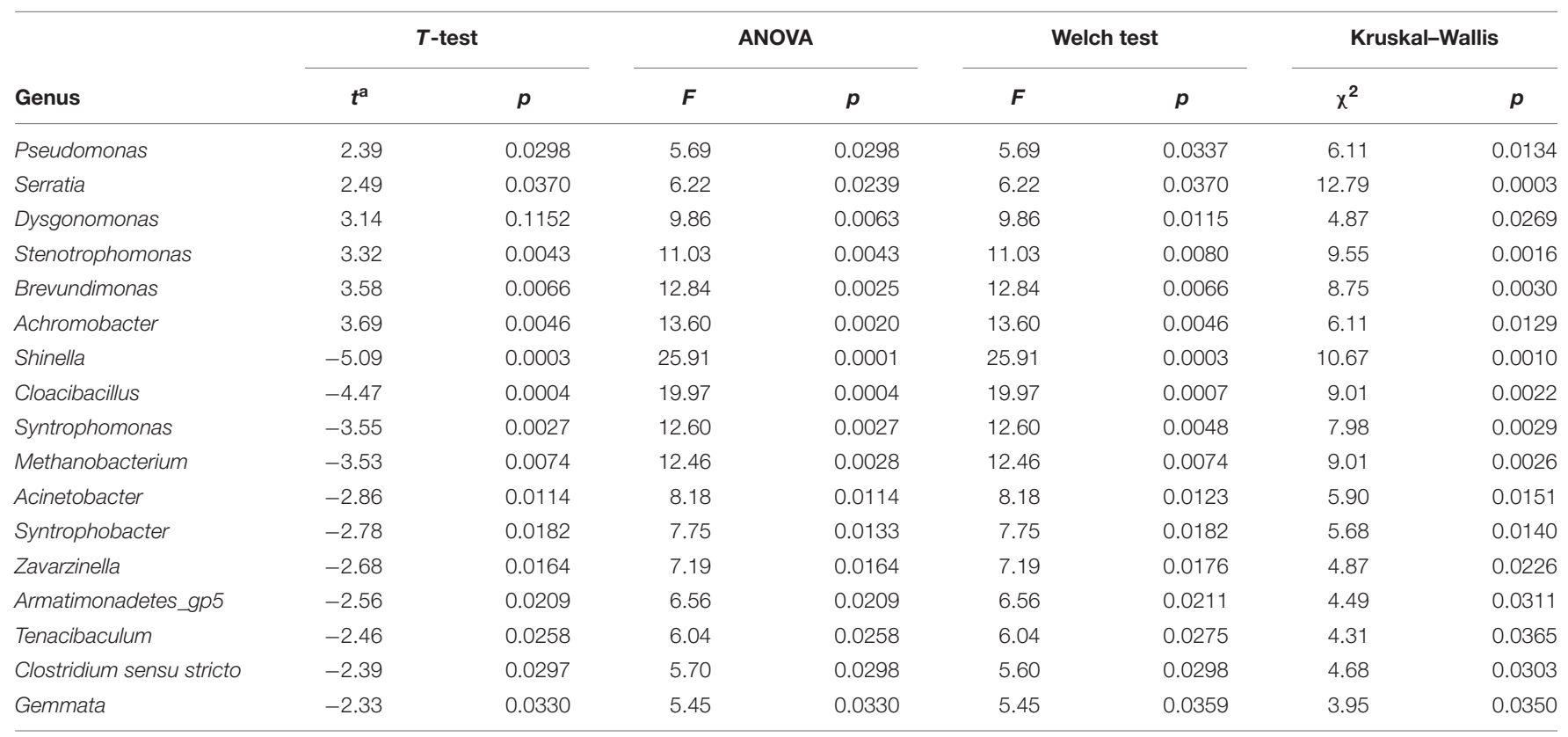

${ }^{a}$ Negative values mean lower abundance in MFC-ABR than $A B R$. 


\section{RESULTS AND DISCUSSION}

\section{Electricity Generation in MFC-ABR}

Electricity generation in all MFC-ABR chambers increased rapidly after inoculation and reached their maximum voltages within 5 days (Figure 1A). Compared with many other MFCs inoculate with anaerobic sludge, MFCs for azo-dyes degradation generally showed more rapid electricity generation (Wang et al., 2009; Gao et al., 2014; Kong et al., 2015) as azo dye reduction products such as aminophenol, phenazine and naphthol can play as electron mediators in electricity generation (van der Zee et al., 2008; Chen et al., 2013). The naphthol generated in AO-7 reduction could be used as an electron mediator by microbes (Chen et al., 2013). The electricity generation of chamber-1 showed the largest variation throughout the experiment probably due to the unstable environments including the dissolved oxygen, $\mathrm{pH}$ and temperature caused by the influents. Moreover, chamber-1 showed the lowest average power density $\left(43.5 \pm 12.7 \mathrm{~mW} / \mathrm{m}^{3}\right)$ while chamber II generated the highest $\left(51.6 \pm 7.2 \mathrm{~mW} / \mathrm{m}^{3}\right.$, Figure 1B). On the other hand, stage I generated the highest average power density while stage III generated the lowest (Figure 1C). These results showed that electricity generation decreased with the increase in AO-7 concentration, suggesting that $\mathrm{AO}-7$ could be used as a more favorable electron acceptor by microorganisms and inhibited electrode reduction in sludge (Yang et al., 2015; Thung et al., 2018).

\section{AO-7 Degradation and Detoxication}

\section{The Enhanced AO-7 Degradation in MFC-ABRs}

On average, $58.3 \pm 7.9 \%$ and $65.5 \pm 10.5 \%$ of AO-7 was reduced in the first chambers of ABRs and MFC-ABRs, respectively (Figure 2A), indicating that chamber-1 played a major role in AO-7 reduction. Furthermore, higher reduction efficiencies were detected in the first chambers of MFC-ABRs than those of ABRs over the experiments (excepted for day 15, 40, and 47). AO-7 showed no significant concentration difference $(<2 \%)$ before and after it flow through the cathode zone $(5 \mathrm{~cm}$ below cathode), suggesting that the AO-7 reduction was mainly occurred in the anodic sludge. In contrast to the inhibition of AO-7 on electricity generation (Figure 1), these results evidenced an enhancement of electricity generation on $\mathrm{AO}-7$ reduction.

Regarding the whole reactor, $\mathrm{ABR}$ and $\mathrm{MFC}-\mathrm{ABR}$ showed similarly AO-7 reduction efficiency $(90.1 \pm 2.1 \%$ vs. $91.4 \pm 1.5 \%$, respectively) in the first two stages (Figure 2B). In stage III, however, the average reduction efficiency in ABR was $85.2 \pm 2.7 \%$, significantly $(p<0.01)$ lower than the first two stages. In contrast, the $\mathrm{AO}-7$ reduction efficiency in MFC-ABR was $91.6 \pm 2.1 \%$ in stage III, comparable to the first two stages, indicating that MFC-ABR maintained a high treatment capacity even with high concentration of AO-7. Therefore, MFC-ABR would be particularly preferred in the treatment of wastewater containing high concentration of azo dyes. The azo dye reduction efficiency in MFC-ABR is comparable to several recently developed systems including ABR-biocatalyzed electrolysis system (ABR-BES), anaerobic
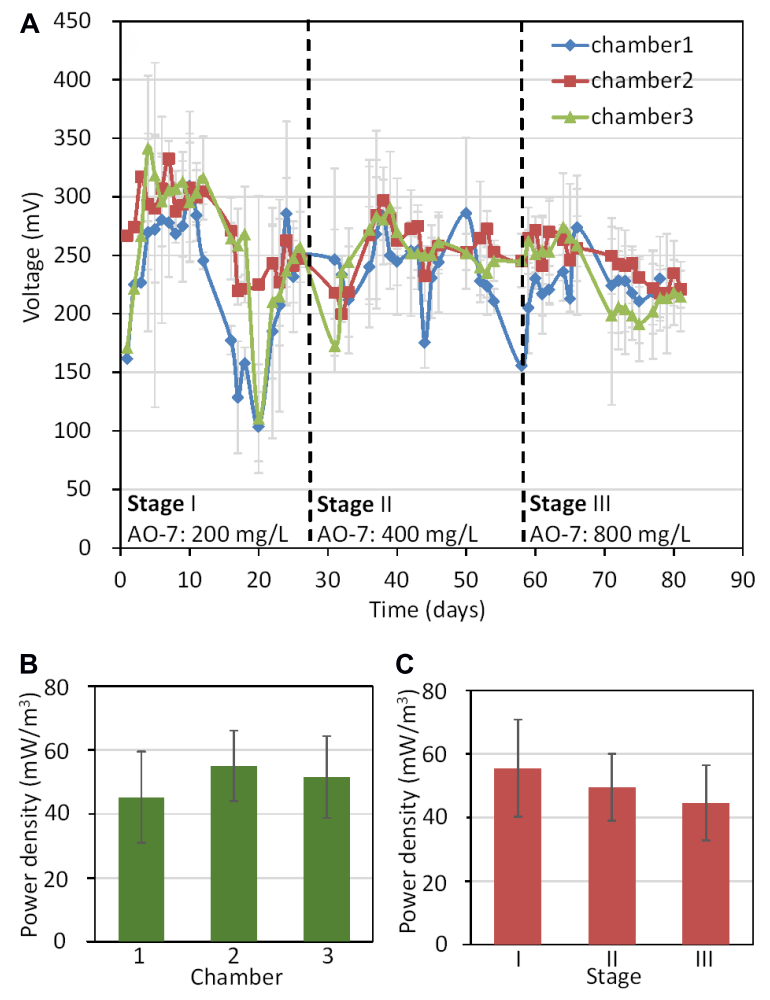

FIGURE 1 | Electricity generation in MFC-ABRs. (A) Voltage curve of the three chambers with different AO-7 concentrations; (B) The average power densities in different chambers; $(\mathbf{C})$ The average power densities in different stages.

sludge reactor-biocatalyzed electrolysis system (ASR-BES) and microbial fuel cell-aerobic reactors (MFC-AR) (Fernando et al., 2013; Cui et al., 2014; Kong et al., 2015; Thung et al., 2018). In contrast to the energy-consuming operations in those reactors (e.g., electrode polarization, aeration), net electricity generation can be obtained in MFC-ABR suggesting an advantage of MFC-ABR for application.

Similar to AO-7 reduction, COD removed mainly occurred in the first chamber in both MFC-ABR and ABR (Supplementary Figure S2). Higher COD removal was observed in MFC-ABR relative to $\mathrm{ABR}$ for all stages. The $\mathrm{CE}$ increased along the flow direction in MFC-ABR: chamber-1 $(1.09 \pm 0.22 \%)<$ chamber- 2 $(5.73 \pm 1.64 \%)<$ chamber-3 $(9.81 \pm 1.19 \%)$ (Supplementary Figure S3). This was consistent with results that the first chamber removed most COD but generated lowest voltage. Moreover, the $\mathrm{CE}$ decreased with the increase of AO-7 concentration: stage-I $(6.89 \pm 0.23 \%)>$ stage-II $(5.44 \pm 0.95 \%)>$ stageIII $(4.29 \pm 0.67 \%)$, probably due to the increasing electron consumption by AO-7. The reported MFC CEs varied largely (from less than 1\% to over 90\%) (Kim et al., 2010, 2011; Zhang et al., 2017). The relative low CE in MFC-ABR indicated that most electrons were consumed by microbial growth, methanogenesis or the other electron acceptors (e.g., AO-7, oxygen, $\mathrm{CO}_{2}$ ) other than microbial anode reduction. 


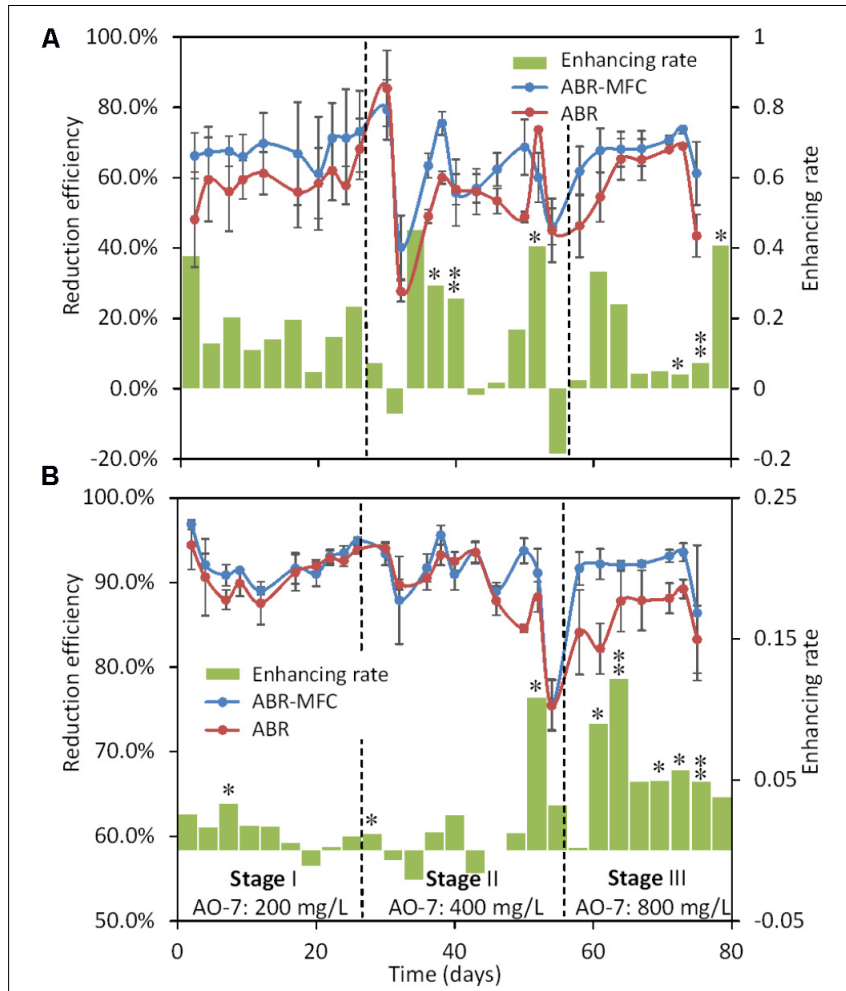

FIGURE 2 | The enhancement of MFC-ABR on AO-7 reduction in chamber-1 (A) and the whole reactor (B). $\left({ }^{*} p<0.05 ;{ }^{*} p<0.01\right)$.

\section{The Degradation Products and Toxicity Evaluation in ABR and MFC-ABR}

Microbial or electrochemical reduction of AO-7 generates 4-aminobenzenesulfonic acid (AA) and 1-amino-2-naphthol (AN) in anaerobic environments (Mu et al., 2009). LC-MS also showed a more rapid removal of AO-7 in each chamber of MFC-ABR than ABR (Figure 3A). AA was detected in all chambers while no AN was detected due to its unstable and auto-oxidizing property in low oxygen environments (Figure 3A) (Kong et al., 2015). Correspondingly, more AA was generated in MFC-ABR and accumulated along the flow direction. AA concentrations in $\mathrm{ABR}(0.58 \pm 0.03 \mathrm{mM})$ or MFC-ABR $(0.67 \pm 0.11 \mathrm{mM})$ could not balance the removal of AO-7 $(2.2 \pm 0.1 \mathrm{mM})$ in both reactors, and the adsorption of AA to sludge has been demonstrated to be negligible (Kong et al., 2015), suggesting that most AA was further degraded in both ABR and MFC-ABR.

Detoxification was the main aim of wastewater treatment. However, toxicity has not been tested in previous BESs for treatment of azo dye-containing wastewaters. In this study, the survival rate of Daphnia magna, one of the most commonly used toxicity tests (Bozich et al., 2017), was used to evaluate the detoxification capacities of ABR and MFC-ABR (Figure 3B). The effluents of all chambers in MFC-ABR at stage III showed higher Daphnia magna survival rate than that of the ABR effluents, suggesting a lower toxicity of MFC-ABR effluents. Both ABR and MFC-ABR showed the highest toxicity in chamber-1 effluents,
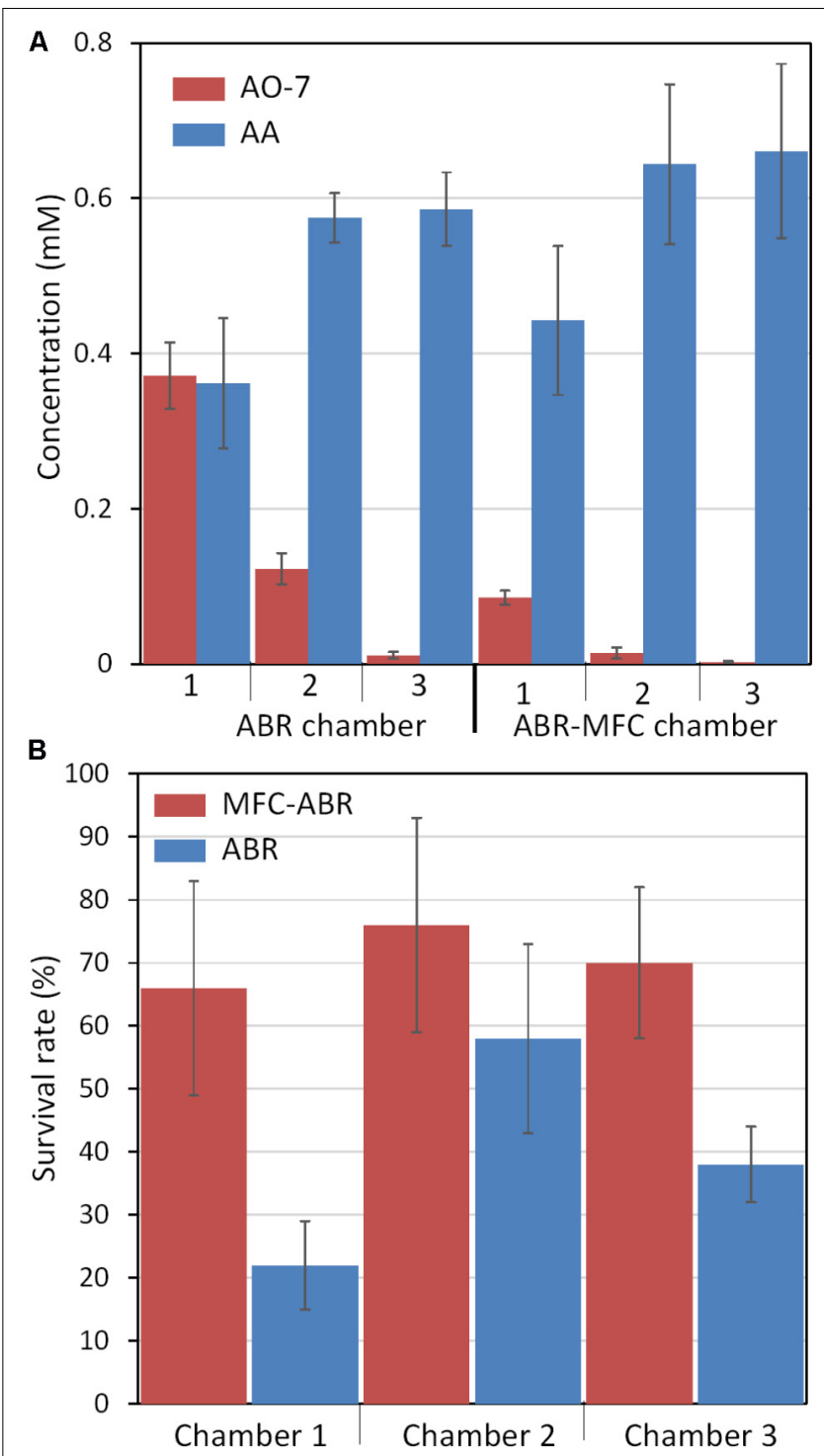

FIGURE 3 | AO-7 reduction products (A) and toxicities (B) in ABRs and MFC-ABRs.

suggesting the necessity of the following two chambers in AO-7 detoxification (Figure 3B). Moreover, toxicity of the effluent was not proportional to the concentration either AO-7 or AA. It can be seen that toxicity is a combined effects of various chemical or biological components in the effluents rather than one or certain contaminants.

\section{Evaluation of the Microbial Activity and Sludge Granule Development}

Both bio-reduction and electrochemical reduction may contribute to the AO-7 reduction in ABR and MFC-ABR. The anode redox potential in MFC-ABR maintained relatively stable at $-0.25 \pm 0.02 \mathrm{~V}$ (vs. SHE) while that of the anode (in open-circuit) in ABR was $-0.3 \pm 0.02 \mathrm{~V}$ (vs. SHE). $\mathrm{Mu}$ et al. (2009) have reported that AO-7 can be slowly 

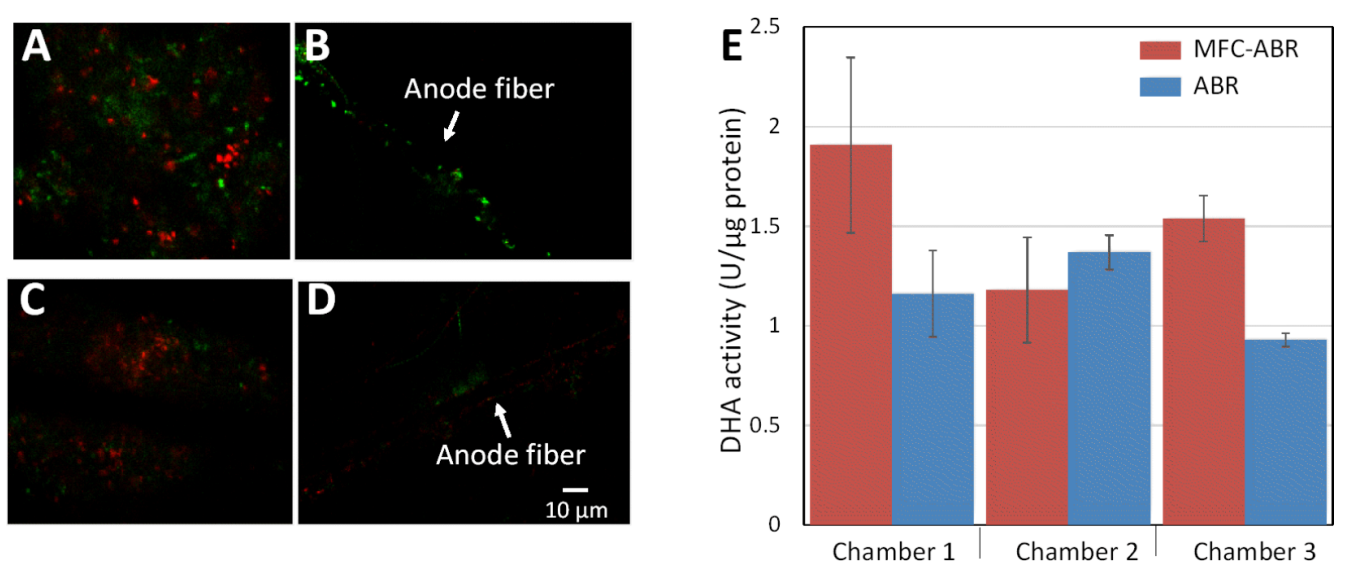

FIGURE 4 | Microbial and DHA activity in chamber 3 of ABR and MFC-ABR. (A,C) Microbial activity of the sludge in MFC-ABR and ABR, respectively; (B,D) microbial activity of the biofilms on carbon fires of MFC-ABR and ABR, respectively. (E) DHA activity of the sludge in different chambers of MFC-ABR and ABR. Green color in (A-D) indicates microbial cells have high metabolism activity and intact cell membrane while red color indicates microbial cells with low metabolism activity and damaged membrane. Scale bar indicates $10 \mu \mathrm{m}$.

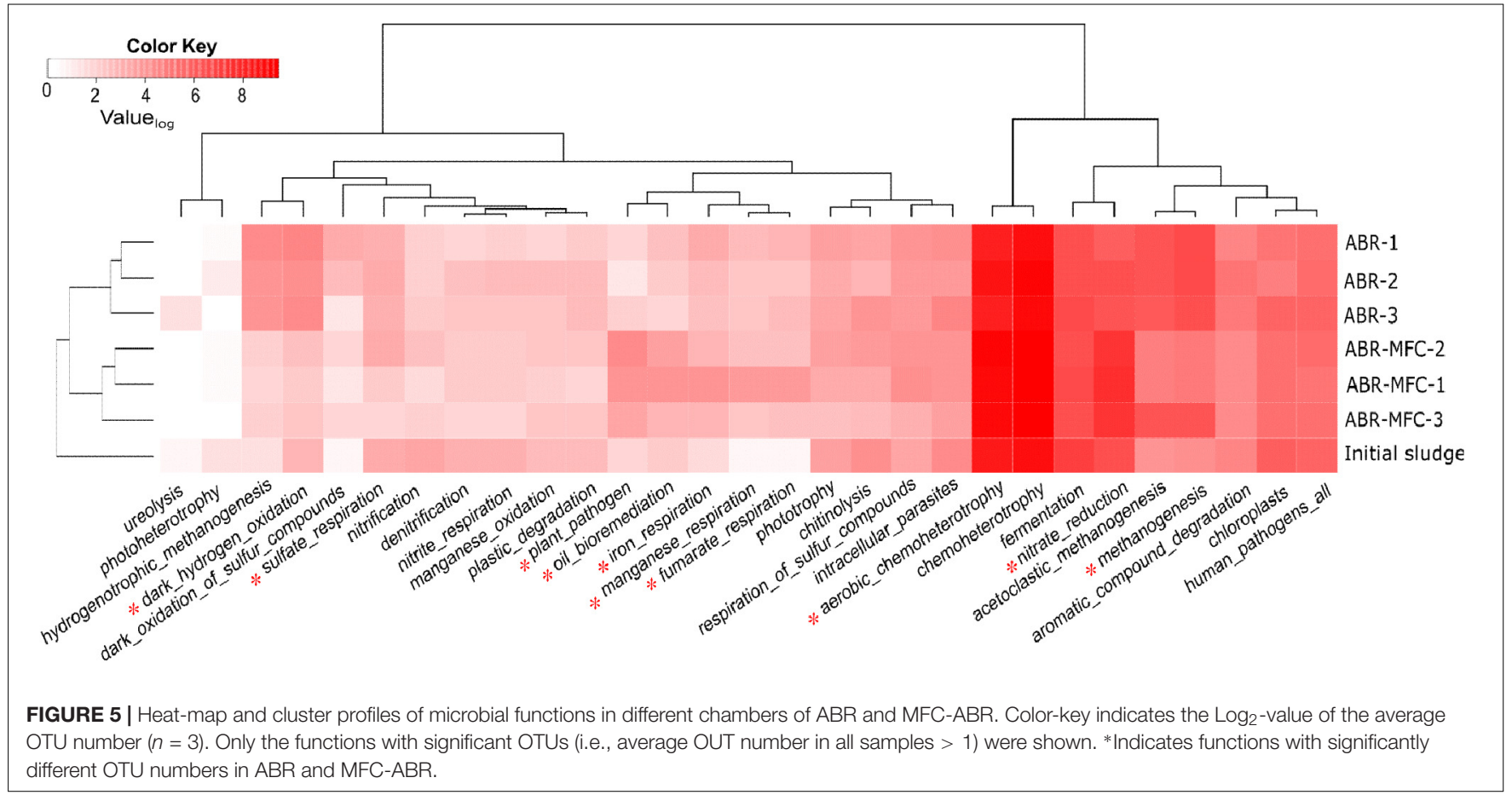

reduced by a graphite electrode at $-0.29 \mathrm{~V}$ (vs. SHE) and the reduction efficiency increased at lower electron potential, indicating that more electrochemical AO-7 reduction occurred in $\mathrm{ABR}$ due to the lower anode potential. Therefore, the higher total AO-7 reduction efficiency in MFC-ABR was mainly caused by bio-reduction rather than electrochemical reduction.

Biomass and bioactivity of the sludge play a key role in contaminant removal in wastewater treatment. Protein-based biomass assessment showed no significant difference among the chambers of ABR and MFC-ABR (Supplementary Figure S4). However, microbial cells in the sludge showed obviously higher viability in MFC-ABR (RV $=0.64 \pm 0.11)$ than that in $\mathrm{ABR}$ $(\mathrm{RV}=0.47 \pm 0.08$, Figure 4). Single layer biofilms $(0-3 \mu \mathrm{m}$ in thickness) were observed on both MFC-ABR and ABR anode fibers, and possible impair on biofilm structure could not be ruled out when the anodes were sampled from sludge. MFC-ABR anode biofilms also showed significantly higher viability $(\mathrm{RV}=0.91 \pm 0.07)$ than that of the $\mathrm{ABR}$ biofilms $(\mathrm{RV}=0.44 \pm 0.05)$. This is consistent to our previous results that electrode could serve as favorable electron acceptor and stimulate the metabolism activity of microbial cells under anaerobic condition (Yang et al., 2015). To further confirm the higher respiratory activity in MFC-ABR, the activity of 


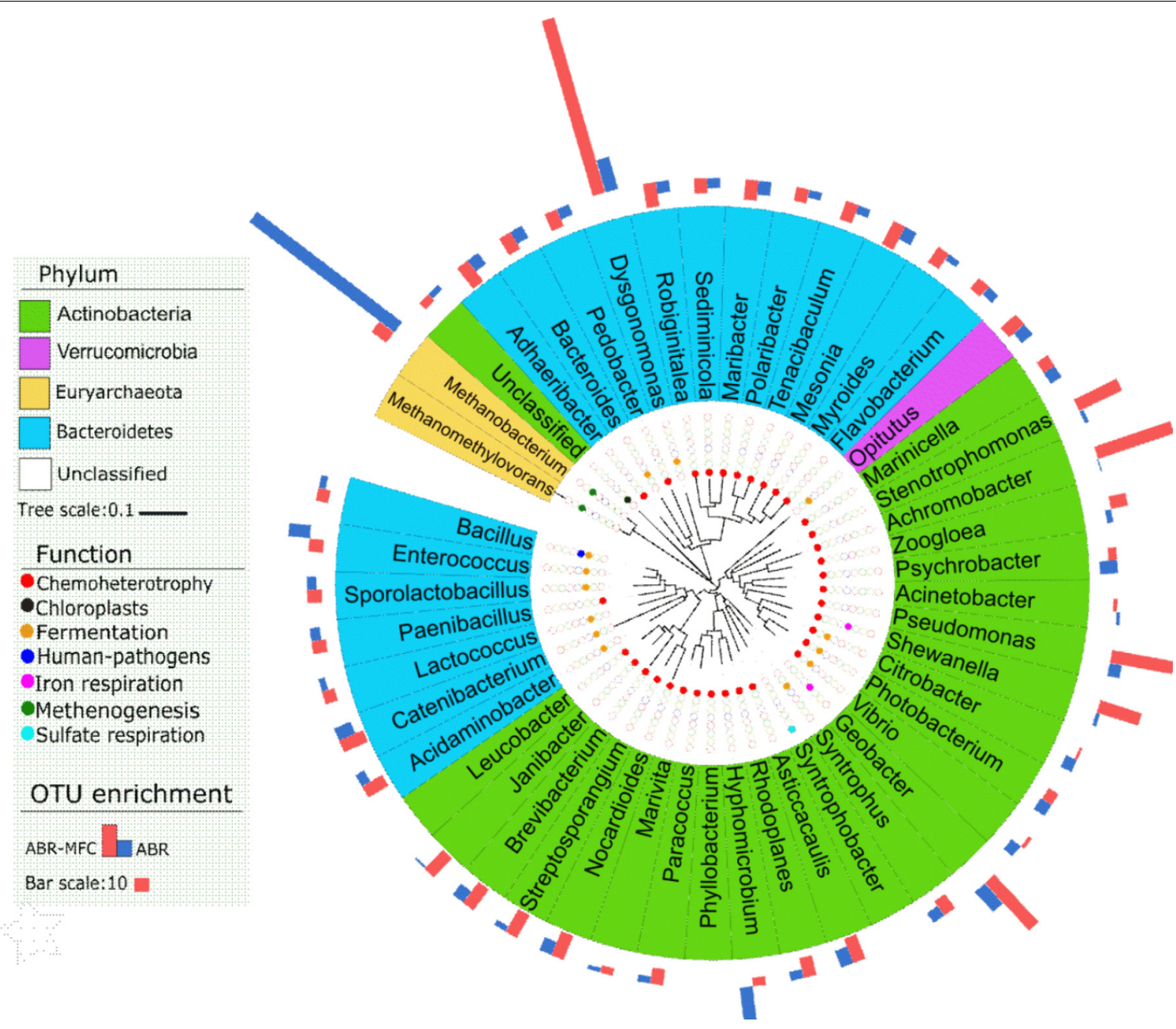

FIGURE 6 | The genera with significantly different $(p<0.05, n=3)$ OTU numbers among ABR, MFC-ABR and initial sludge and their annotated functions in FAPROTAX. The background color of each genus indicate its phylum. The circles filled with different colors indicate the functions of the corresponding genus. The bars in the outmost layer indicate the enhanced-fold of the genus in MFC-ABR and ABR, relative to the genus in initial sludge.

DHA, an essential component in bacterial electron transfer (Stolz, 2001; Shi et al., 2009), was measured. DHA activities in chamber- 1 and chamber-3 of MFC-ABR were significantly higher than that of ABR (Figure 4), indicating a higher electron transfer activity in these cambers. It should be noted that DHA is a group of intracellular redox enzymes. Higher DHA activity accounts for higher intracellular electron transfer activity (e.g., AO-7 reduction) but cannot ensure a higher EET activity, because all microbes have DHA but only a minor portion of microbes have EET capability (Koch and Harnisch, 2016).

The development of sludge granule is also an important factor affecting the treatment capacity in the reactors. The numbers of big sludge granules (diameter $\geq 100 \mu \mathrm{m}$ ) in all chambers of MFC-ABR were significantly higher than that in ABR (Supplementary Figure S5). Sludge granules with a diameter over $150 \mu \mathrm{m}$ were detected in all MFC-ABR chambers but not in ABR. The results suggested that the growth of sludge particles was also improved by anode respiration which might contribute to higher AO-7 removal efficiency in MFC-ABR.

\section{Microbial Community Structure and Function The Changes of Microbial Community Structure}

A total of 261, 162 effective reads were retrieved after trimming the adapters, barcodes, primers and filtering the low quality reads including chimeras. The number of OTUs at a cutoff level of 3\% ranged from 1624 to 1983 among different samples with slightly higher numbers observed in ABR (1859 \pm 115$)$ than in MFC-ABR (1775 \pm 88 ). Accordingly, the alpha diversity estimated by the Chaol and Shannon indexes in MFC-ABR was significantly lower than ABR $(p<0.05)$. The first MFC-ABR chamber showed significantly higher diversity than the following two chambers while no difference was observed among ABR chambers (Supplementary Table S1). Principal Coordinate Analysis (PCoA) based on Bray-Curtis distance of OTUs showed 
that the samples could be grouped into MFC-ABR and ABR clustered by PCoA1 axes (Supplementary Figure S6). The above results indicated a significant role of electricity generation in shaping microbial community structure. RDA was used to evaluate the associations between reactor variables and the communities. The first two axes explained 66.3 and $45.8 \%$ of the microbial community variations in MFC-ABR and $A B R$, respectively. DO in MFC-ABR $\left(r^{2}=0.66, p=0.04\right)$ while ORP $\left(r^{2}=0.5466, p=0.07\right)$ and removal efficiency $\left(r^{2}=0.64, p=0.03\right)$ in ABR were the major drivers affecting community structure and compositions.

With RDP Classifier at an $80 \%$ threshold, all effective microbial sequences were assigned to different taxa levels. Among a total of 28 detected phyla (Supplementary Figure S7), Proteobacteria was the most abundant phylum that accounted for $43.7 \pm 7.3 \%$ and $33.7 \pm 7.4 \%$ of total microbial sequences in MFC-ABR and ABR, respectively. Firmicutes $(15.7 \pm 4.0 \%$ and $18.5 \pm 3.4 \%)$, Bacteroidetes $(8.0 \pm 0.7 \%$ and $7.8 \pm 1.7 \%)$, Chloroflexi (10.0 $\pm 3.1 \%$ and $12.1 \pm 3.3 \%)$, Actinobacteria (2.5 $\pm 0.5 \%$ and $3.1 \pm 0.5 \%)$ also dominated communities in both ABR and MFC-ABR. Within phylum Proteobacteria, $\gamma$-proteobacteria $(21.1 \pm 4.3 \%)$ and $\beta$-proteobacteria $(8.2 \pm 2.6 \%)$ had significantly higher abundances in MFC-ABR than ABR $(14.3 \pm 6.1 \%, 4.7 \pm 1.2 \%)$.

At the genus level, Pseudomonas (6.7 - 9.9\%), Sporolactobacillus $(3.2$ - 4.7\%), Clostridium sensu stricto (1.7 - 3.9\%), Lewinella (1.5 - 1.9\%) and Longilinea (1.1 - 1.6\%) showed $>1 \%$ abundance in all MFC-ABR chambers, representing the core consortia in MFC-ABR. Compared to the ABR community, the abundances of seven genera were higher whereas nine genera were lower than those in the MFC-ABR $(P<0.05$, Table 1). Among genera enriched in the MFC-ABR, Pseudomonas showed increasing abundance along the flow direction, which was consistent with its versatile capacities including azo dye reduction, electricity generation, aromatic compounds biodegradation and fermentation (Galai et al., 2015). The other MFC-ABR-enriched genera have also shown capacities such as EET (Serratia, Stenotrophomonas), azo-dye reduction (Stenotrophomonas) or aromatic compounds degradation (Dysgonomonas, Brevundimonas, and Achromobacter) (Jin et al., 2015; Paul et al., 2015; Hao et al., 2016), and most of them showed increasing abundances along with the flow direction. On the other hand, the abundances of genera associated with fermentation and methanogenesis were lower in MFC-ABR relative to ABR, such as Acinetobacter, Cloacibacillus and Methanobacterium (Krivushin et al., 2010; Lovley, 2011; Looft et al., 2013).

\section{The Prediction of Microbial Community Function}

The FAPROTAX database has been proposed as a tool to predict possible environmental functions of microbial communities (Louca et al., 2016). FAPROTAX predicted 92 functions of OTUs in the sludge samples and 30 (with average OTU $>1$ ) of them were shown in Figure 5. The functional compositions of ABR chambers and MFC-ABR chambers were well separated, indicating functional diversification in the two types of reactor. Chemoheterotrophy $(34.9 \pm 1.7 \%)$ was the predominant process predicted by FAPROTAX in both reactors, followed by nitrate reduction $(7.4 \pm 2.3 \%)$ and fermentation (6.6 $\pm 1.6 \%)$. Ten functions showed significantly different OTU abundances between ABR and MFC-ABR. Seven of them, including oil bioremediation, aerobic chemoheterotrophy, plant pathogen, iron respiration, manganese respiration, fumarate respiration and nitrate respiration were enhanced in MFC-ABR relative to $\mathrm{ABR}$ while the other three (hydrogen-oxidization, sulfate respiration and methanogenesis) were suppressed. Consistent with RDA and community analyses, the chamber-specific functional structure also suggested a sequentially segregated biochemical processes in both ABR and MFC-ABR. Compared with the enhanced nitrate respiration, sulfate respiration was gradually decreased from chamber 1-3 in MFC-ABR, indicating that the electricity generation suppressed sulfate respiration but co-existed with nitrate respiration (Figure 5). Moreover, the abundances of most electricity-stimulated respirations including iron-, manganese-, fumarate- and nitrate-respirations showed the highest abundances in the first chamber and decreased along the flow direction, suggesting that those functions were not only enhanced by electricity generation but also by azo reduction. The profiles of those anaerobic respirations coincided with the higher microbial viability and DHA activity shown in Figure 4, as the DHA and c-type cytochromes were commonly shared as key electron transfer components in those respirations (Shi et al., 2009). Anaerobic respirations (except for sulfate reduction) and oil-bioremediation, mainly aromatics degradation, always showed consistent abundances in both ABR and MFC-ABR, indicating a synergistic relationship among the bacterial respiration and aromatics biodegradation under anaerobic conditions.

To identify the key bacteria contributing to the functional differences in Figure 5, FAPROTAX identified 48 genera that shared by ABR and MFC-ABR but differ in their relative abundances $(p<0.05)$ (Figure 6). Those genera involved in eight functions, including iron respiration (2 genera), fermentation (13 genera), methenogenesis (2 genera), chemoheterotrophy (32 genera), human pathogens (1 genus), sulfate respiration (1 genus), photosynthesis (1 genus). The enhancements of Pseudomonas, Stenotrophomonas, Achromobacter, Dysgonomonas in MFC-ABR were also noted by FAPROTAX. Two typical electricity generating bacteria, Shewanella and Geobacter (Shi et al., 2009), were predicted to play key roles in the enhancement of iron respiration in MFC-ABR. In contrast, 11 fermentation bacteria and two methenogenesis archaea (Methanobacterium and Methanomethylovorans) were the key members contributing to the enrichment of fermentation and methenogenesis in ABR. It should be noted that the other significantly differed functions between $\mathrm{ABR}$ and MFC-ABR were caused by bacteria without significant abundances or not included in FAPROTAX.

Community composition and functional predication suggested that the low energy-generating processes such as fermentation, methenogenesis and sulfate reduction were suppressed by electricity generation due to the relative high redox potential of MFC anode. Previous transcriptomic analyses have showed that electron acceptor redox potential could 
alter bacterial metabolism pathway (Lian et al., 2016). Our Geochip data of sediment MFC showed that anodes could enhance the functional genes such as nitrate reductase, reductive dehalogenase, aromatic degradation genes, partially supporting the community composition profiles in this study (Yang et al., 2013). The co-enriched versatile bacteria for respiration and biodegradation by microbial anode reduction might also contribute to the higher AO-7 degradation and detoxification in MFC-ABR. However, it should be noted that FAPROTAX prediction only provided possible microbial functions, further evidences such as gene-expression, comprehensive chemical analyses are needed to test the reliability of the prediction.

\section{CONCLUSION}

Microbial anode respiration could significantly enhanced AO-7 degradation and detoxication in MFC-ABR compared with ABR, especially under high AO-7 concentration, which could be attributed to the higher microbial activity, dehydrogenase activity and larger sludge granule size in MFC-ABR. Moreover, microbial anode respiration dramatically reshaped the community structure and decreased the diversity of the microbial communities. Pseudomonas was the most abundant genus enriched by electricity generation. In contrast to the suppressed bacteria capable of methanogenesis and sulfate respiration, the co-enriched bacteria with versatile respiration (e.g., EET, fumarate and nitrate respiration) and biodegradation capabilities provided useful information to understand the enhanced degradation and detoxication of $\mathrm{AO}-7$ by microbial anode respiration.

\section{REFERENCES}

Bozich, J., Hang, M. M., Hamers, R., and Klaper, R. (2017). Core chemistry influences the toxicity of multicomponent metal oxide nanomaterials, lithium nickel manganese cobalt oxide, and lithium cobalt oxide to Daphnia magna. Environ. Toxicol. Chem. 36, 2493-2502. doi: 10.1002/etc.3791

Chen, B. Y., Hsueh, C. C., Liu, S. Q., Ng, I. S., and Wang, Y. M. (2013). Deciphering mediating characteristics of decolorized intermediates for reductive decolorization and bioelectricity generation. Bioresour. Technol. 145, 321-325. doi: 10.1016/j.biortech.2012.12.164

Cui, D., Guo, Y. Q., Lee, H. S., Wu, W. M., Liang, B., Wang, A. J., et al. (2014). Enhanced decolorization of azo dye in a small pilot-scale anaerobic baffled reactor coupled with biocatalyzed electrolysis system (ABR-BES): a design suitable for scaling-up. Bioresour. Technol. 163, 254-261. doi: 10.1016/ j.biortech.2014.03.165

de Aragao Umbuzeiro, G., Freeman, H. S., Warren, S. H., de Oliveira, D. P., Terao, Y., Watanabe, T., et al. (2005). The contribution of azo dyes to the mutagenic activity of the Cristais river. Chemosphere 60, 55-64. doi: 10.1016/ j.chemosphere.2004.11.100

Fernando, E., Keshavarz, T., and Kyazze, G. (2013). Simultaneous co-metabolic decolourisation of azo dye mixtures and bio-electricity generation under thermophillic and saline conditions by an adapted anaerobic mixed culture in microbial fuel cells. Bioresour. Technol. 127, 1-8. doi: 10.1016/j.biortech.2012. 09.065

Galai, S., de los Rios, A. P., Hernandez-Fernandez, F. J., Kacem, S. H., Ramirez, F. M., and Quesada-Medina, J. (2015). Microbial fuel cell application for azoic dye decolorization with simultaneous bioenergy production using Stenotrophomonas sp. Chem. Eng. Technol. 38, 1511-1518. doi: 10.1002/ceat. 201400608

\section{AUTHOR CONTRIBUTIONS}

YY and MX designed the study. YY and OL operated the experiments. YY, OL, MX, GK, BW, XL, EL, and JL analyzed and discussed the results. YY, OL, and FL wrote the paper. All the authors agree to be accountable for the content of the work.

\section{FUNDING}

This research was supported by the National Natural Science Foundation of China (51422803, 31570111, 51678163, U1701243), Guangdong Provincial Science and Technology Project GDAS' Special Project of Science and Technology Development (2017GDASCX-0401, 2017GDASCX-0403, 2016GDASRC-0208, 2017GDASCX0102), (2014TQ01Z116, 2014TX01Z038, 2016B070701017), Guangdong Provincial Natural Science Foundation (2014A030308019, 2016A030306021), Guangdong Ocean and Fishery Administration Project (A201601D01), Pearl River S\&T Nova Program of Guangzhou (201610010090), and Open Project of Key Laboratory of Environmental Biotechnology (CAS kf2016003).

\section{SUPPLEMENTARY MATERIAL}

The Supplementary Material for this article can be found online at: https://www.frontiersin.org/articles/10.3389/fmicb. 2018.02117/full\#supplementary-material

Gao, C., Wang, A., Wu, W. M., Yin, Y., and Zhao, Y. G. (2014). Enrichment of anodic biofilm inoculated with anaerobic or aerobic sludge in single chambered air-cathode microbial fuel cells. Bioresour. Technol. 167, 124-132. doi: 10.1016/ j.biortech.2014.05.120

Hao, T., Zhang, B. G., Cheng, M., and Feng, C. P. (2016). Effects of various organic carbon sources on simultaneous $\mathrm{V}(\mathrm{V})$ reduction and bioelectricity generation in single chamber microbial fuel cells. Bioresour. Technol. 201, 105-110. doi: 10.1016/j.biortech.2015.11.060

Jiang, X., Shen, J., Xu, K., Chen, D., Mu, Y., Sun, X., et al. (2018). Substantial enhancement of anaerobic pyridine bio-mineralization by electrical stimulation. Water Res. 130, 291-299. doi: 10.1016/j.watres.2017. 12.005

Jin, D., Kong, X., Li, Y., Bai, Z., Zhuang, G., Zhuang, X., et al. (2015). Biodegradation of di-n-butyl phthalate by Achromobacter sp. isolated from rural domestic wastewater. Int. J. Environ. Res. Public Health 12, 13510-13522. doi: 10.3390/ijerph121013510

Kim, J. R., Premier, G. C., Hawkes, F. R., Rodríguez, J., Dinsdale, R. M., and Guwy, A. J. (2010). Modular tubular microbial fuel cells for energy recovery during sucrose wastewater treatment at low organic loading rate. Bioresour. Technol. 101, 1190-1198. doi: 10.1016/j.biortech.2009. 09.023

Kim, K. Y., Chae, K. J., Choi, M. J., Ajayi, F. F., Jang, A., Kim, C. W., et al. (2011). Enhanced coulombic efficiency in glucose-fed microbial fuel cells by reducing metabolite electron losses using dual-anode electrodes. Bioresour. Technol. 102, 4144-4149. doi: 10.1016/j.biortech.2010.12.036

Koch, C., and Harnisch, F. (2016). What is the essence of microbial electroactivity? Front. Microbiol. 7:1890. doi: 10.3389/Fmicb.2016.01890

Kong, F., Wang, A., and Ren, H. Y. (2015). Improved azo dye decolorization in an advanced integrated system of bioelectrochemical module with surrounding 
electrode deployment and anaerobic sludge reactor. Bioresour. Technol. 175, 624-628. doi: 10.1016/j.biortech.2014.10.091

Krivushin, K. V., Shcherbakova, V. A., Petrovskaya, L. E., and Rivkina, E. M. (2010). Methanobacterium veterum sp nov., from ancient Siberian permafrost. Int. J. Syst. Evol. Microbiol. 60, 455-459. doi: 10.1099/ijs.0.011205-0

Li, Z., Zhang, X. W., Lin, J., Han, S., and Lei, L. (2010). Azo dye treatment with simultaneous electricity production in an anaerobic-aerobic sequential reactor and microbial fuel cell coupled system. Bioresour. Technol. 101, 4440-4445. doi: 10.1016/j.biortech.2010.01.114

Lian, Y., Yang, Y., Guo, J., Wang, Y., Li, X., Fang, Y., et al. (2016). Electron acceptor redox potential globally regulates transcriptomic profiling in Shewanella decolorationis S12. Sci. Rep. 6:31143. doi: 10.1038/srep31143

Liu, H., Hu, T. J., Zeng, G. M., Yuan, X. Z., Wu, J. J., Shen, Y., et al. (2013). Electricity generation using p-nitrophenol as substrate in microbial fuel cell. Int. Biodeterior. Biodegrad. 76, 108-111. doi: 10.1016/j.watres.2009. 04.004

Logan, B. E., Hamelers, B., Rozendal, R. A., Schrorder, U., Keller, J., Freguia, S., et al. (2006). Microbial fuel cells: methodology and technology. Environ. Sci. Technol. 40, 5181-5192. doi: 10.1021/Es0605016

Looft, T., Levine, U. Y., and Stanton, T. B. (2013). Cloacibacillus porcorum sp. nov., a mucin-degrading bacterium from the swine intestinal tract and emended description of the genus Cloacibacillus. Int. J. Syst. Evol. Microbiol. 63, 1960-1966. doi: 10.1099/ijs.0.044719-0

Louca, S., Jacques, S. M. S., Pires, A. P. F., Leal, J. S., Srivastava, D. S., Parfrey, L. W., et al. (2016). High taxonomic variability despite stable functional structure across microbial communities. Nat. Ecol. Evol. 1:15. doi: 10.1038/s41559-0160015

Lovley, D. R. (2011). Live wires: direct extracellular electron exchange for bioenergy and the bioremediation of energy-related contamination. Energy Environ. Sci. 4, 4896-4906. doi: 10.1039/C1ee02229f

Mu, Y., Rabaey, K., Rozendal, R. A., Yuan, Z., and Keller, J. (2009). Decolorization of azo dyes in bioelectrochemical systems. Environ. Sci. Technol. 43, 5137-5143. doi: 10.1021/es900057f

Paul, D., Kazy, S. K., Das Banerjee, T., Gupta, A. K., Pal, T., and Sar, P. (2015). Arsenic biotransformation and release by bacteria indigenous to arsenic contaminated groundwater. Bioresour. Technol. 188, 14-23. doi: 10.1016/j. biortech.2015.02.039

Pham, H., Boon, N., Marzorati, M., and Verstraete, W. (2009). Enhanced removal of 1,2-dichloroethane by anodophilic microbial consortia. Water Res. 43, 2936-2946. doi: 10.1016/j.watres.2009.04.004

Shi, L., Richardson, D. J., Wang, Z. M., Kerisit, S. N., Rosso, K. M., Zachara, J. M., et al. (2009). The roles of outer membrane cytochromes of Shewanella and Geobacter in extracellular electron transfer. Environ. Microbiol. Rep. 1, 220-227. doi: 10.1111/j.1758-2229.2009.00035.x

Solanki, K., Subramanian, S., and Basu, S. (2013). Microbial fuel cells for azo dye treatment with electricity generation: a review. Bioresour. Technol. 131, 564-571. doi: 10.1016/j.biortech.2012.12.063

Solis, M., Solis, A., Perez, H. I., Manjarrez, N., and Flores, M. (2012). Microbial decolouration of azo dyes: a review. Proc. Biochem. 47, 1723-1748. doi: 10.1016/ j.procbio.2012.08.014

Stolz, A. (2001). Basic and applied aspects in the microbial degradation of azo dyes. Appl. Microbiol. Biotechnol. 56, 69-80. doi: 10.1007/s002530100686

Sun, J., Hu, Y. Y., Bi, Z., and Cao, Y. Q. (2009). Simultaneous decolorization of azo dye and bioelectricity generation using a microfiltration membrane aircathode single-chamber microbial fuel cell. Bioresour. Technol. 100, 3185-3192. doi: 10.1016/j.biortech.2009.02.002
Sun, W., Xia, C. Y., Xu, M. Y., Guo, J., and Sun, G. P. (2017). Seasonality affects the diversity and composition of bacterioplankton communities in dongjiang river, a drinking water source of Hong Kong. Front. Microbiol. 8:1644. doi: 10.3389/fmicb.2017.01644

Thung, W. E., Ong, S. A., Ho, L. N., Wong, Y. S., Ridwan, F., Kaur Lehl, H., et al. (2018). Biodegradation of acid orange 7 in a combined anaerobic-aerobic up-flow membrane-less microbial fuel cell: mechanism of biodegradation and electron transfer. Chem. Eng. J. 336, 397-405. doi: 10.1016/j.cej.2017. 12.028

van der Zee, F. P., Lettinga, G., and Field, J. A. (2008). The role of (auto)catalysis in the mechanism of an anaerobic azo reduction. Water Sci. Technol. 42, 301-308.

Wang, X., Feng, Y. J., Ren, N. Q., Wang, H. M., Lee, H., Li, N., et al. (2009). Accelerated start-up of two-chambered microbial fuel cells: effect of anodic positive poised potential. Electrochim. Acta 54, 1109-1114. doi: 10.1016/j. electacta.2008.07.085

Xu, F., Cao, F., Kong, Q., Zhou, L., Yuan, Q., Zhu, Y., et al. (2018a). Electricity production and evolution of microbial community in the constructed wetlandmicrobial fuel cell. Chem. Eng. J. 339, 479-486. doi: 10.1016/j.cej.2018. 02.003

Xu, H., Tong, N., Huang, S., Zhou, S., Li, S., Li, J., et al. (2018b). Degradation of 2,4,6-trichlorophenol and determination of bacterial community structure by micro-electrical stimulation with or without external organic carbon source. Bioresour. Technol. 263, 266-272. doi: 10.1016/j.biortech.2018.05.015

Xu, M., Zhang, Q., Xia, C., Zhong, Y., Sun, G., Guo, J., et al. (2014). Elevated nitrate enriches microbial functional genes for potential bioremediation of complexly contaminated sediments. ISME J. 8, 1932-1944. doi: 10.1038/ismej. 2014.42

Yang, Y., Xiang, Y., Sun, G., Wu, W. M., and Xu, M. (2015). Electron acceptordependent respiratory and physiological stratifications in biofilms. Environ. Sci. Technol. 49, 196-202. doi: 10.1021/es504546g

Yang, Y., Xu, M., He, Z., Guo, J., Sun, G., and Zhou, J. (2013). Microbial electricity generation enhances decabromodiphenyl ether (BDE-209) degradation. PLoS One 8:e70686. doi: 10.1371/journal.pone.0070686

Yin, J., Tan, X. J., Ren, N. Q., Cui, Y. B., and Tang, L. (2005). Evaluation of heavy metal inhibition of activated sludge by TTC and INT-electron transport system activity tests. Water Sci. Technol. 52, 231-239.

Zhang, D., Li, Z., Zhang, C., Zhou, X., Xiao, Z., Awata, T., et al. (2017). Phenoldegrading anode biofilm with high coulombic efficiency in graphite electrodes microbial fuel cell. J. Biosci. Bioeng. 123, 364-369. doi: 10.1016/j.jbiosc.2016. 10.010

Zhao, Y., Li, Z., Ma, J., Yun, H., Qi, M., Ma, X., et al. (2018). Enhanced bioelectroremediation of a complexly contaminated river sediment through stimulating electroactive degraders with methanol supply. J. Hazard. Mat. 349, 168-176. doi: 10.1016/j.jhazmat.2018.01.060

Conflict of Interest Statement: The authors declare that the research was conducted in the absence of any commercial or financial relationships that could be construed as a potential conflict of interest.

Copyright (C) 2018 Yang, Luo, Kong, Wang, Li, Li, Li, Liu and Xu. This is an openaccess article distributed under the terms of the Creative Commons Attribution License (CC BY). The use, distribution or reproduction in other forums is permitted, provided the original author(s) and the copyright owner(s) are credited and that the original publication in this journal is cited, in accordance with accepted academic practice. No use, distribution or reproduction is permitted which does not comply with these terms. 